\title{
VANET based Intelligent TransportationSystem using Li-Fi technology
}

\author{
Bildass Santhosam. $\mathrm{I}^{1}$, Divya. $\mathrm{D}^{2}$, Priyanka. $\mathrm{R}^{3}$ \\ Assistant professor, ECE, CSICE, The Nilgiris, India ${ }^{1}$ \\ UG student, ECE, CSICE, The Nilgiris, India ${ }^{2}$ \\ UG student, ECE, CSICE, The Nilgiris, India ${ }^{3}$
}

\begin{abstract}
VANET (Vehicular Ad-hoc Network) is a wireless network in vehicle for Intelligent Transportation System (ITS).In thispaper, we propose a mechanism toprevent accidents due to sleepiness and alcohol consumption of the driver. Vehicle to Vehicle (V2V) communication is the most effective solutionwe have used in order to prevent accidents using the Li-Fi technology.
\end{abstract}

Keywords: VANET, V2V, ITS, Li-Fi.

\section{Introduction}

Vehicular Ad-hoc Network (VANET) is being used for communication between Vehicles, not only for communication purpose, it is also structured for traffic controlling, Navigation, and other application in VANET. Intelligent transportation systems (ITS) are advanced applications which, without embodying intelligence as such, aim to provide innovative services relating to different modes of transport and traffic management and enable various users to be better informed and make safer, more coordinated, and 'smarter' use of transport networks. VANET is a technology that uses moves vehicles as nodes in a network to create a mobile network. It is a sub-class of Mobile Ad-hoc Network(MANET). Each vehicle is equipped with a communication device can act not only as a communication node,but also as a wireless router and it does not require a service provider.The ad-hoc Networkis a temporary networkthat is created by the absence of any centralized or pre-established infrastructure. VANET turns every participating vehicle into a wireless router or node, allowing vehicle, approximately 100 to 300 meters of each Other to connect and, in turn, create a network with a wide range. As vehicle falls out of the signal range and drop out of the network, other vehicles can join in, connecting vehicles to one another so that a mobile Internet is created. It is estimated that the first systems that will integrate this technology are police and fire vehicles to communicate with each other for safety purposes.

Li-Fi technology is a groundbreaking light-based communication technology, which makes use of light waves instead of radio technology to deliver data. Using the visible light spectrum, Li-Fi technology can transmit data and unlock capacity, which is 10,000 times greater than that available within the radio spectrum. The visible light spectrum is plentiful, free and unlicensed, mitigating the radio frequency spectrum crunch effect. Most of the intelligent transportation system is used to monitor the system functions such as antilock brakes, speed sensor, vehicle position and other automated system are present in the sports and other luxury vehicles only, but these vehicles are not affordable to everyone. So a system needs to be developed which can be implemented in every vehicle.

\section{Objective}

The method of V2V communicationis used to prevent road accidents by using the data collected by thesensors. Toestablish high speed connection for data transmission (in case of disaster problem), Li-Fi is used because it is fast and moreeffective.

\section{Proposed System}

In our paper, we make use of three types of sensors 1) UltrasonicSensor 2)AlcoholSensor 3)Eye blink Sensor

(i) Ultrasonic sensor:

An ultrasonic sensor is a collision avoidance system and used to measure the distance between vehicles or obstacles which is placed within a vehicle to warn its driver of any dangers that may lie ahead on the road. Some of the dangers that these sensors can pick up on include how close the vehicle is to other vehicles surrounding to it, how much its speed needs to be reduced while going around a curve, and how close the vehicle is going off the road. The system uses sensors that send and receive signals from thinks like other vehicles, obstacles in the road, traffic signals and the central database are placed within the vehicle and tell it of any traffic precautions.

\section{(ii) Alcohol Sensor}

If the person driving thevehicle has consumed alcohol, then the alcohol detection is done by the sensor. Sensor gives this signal to anAnalog to DigitalConverter (ADC) which converts the analog signal to digital signal and 
then to the microcontroller. Microcontroller gives a high pulse to the buzzer circuit and the buzzer is turned on. At the same time a relay is turned off. Due to this the ignition of the vehicle is deactivated. The information collected from the sensor is beingtransmitted to neighboring vehicles through V2V communication using VLC transmitter. Alcohol Detection System present inthe vehicle provides an automatic safety system for vehicles and other vehicles as well.

(iii)Eyeblink Sensor

The Eyeblink Sensor is being fitted in the dashboard of the vehicle. It continuously monitors the movement of the eye, if the closure of the eye is detected, then the microcontroller gives a high pulse to the buzzer circuit and the buzzer is turned on. So by the buzzer sound the driver would be alerted.

\section{System Design}

The system requires a transmitter and a receiver in each vehicle in both rear and front sides of the vehicle.The system design is being divided into two sections(i) transmission (ii) receiver section.

The transmitter section consists of the sensors, ADC, Microcontroller (PIC16F877A), UART, Buzzer, VLC Transmitter and LCD display. The receiver section consists of the VLC receiver, TTL to UART and the LCD display.

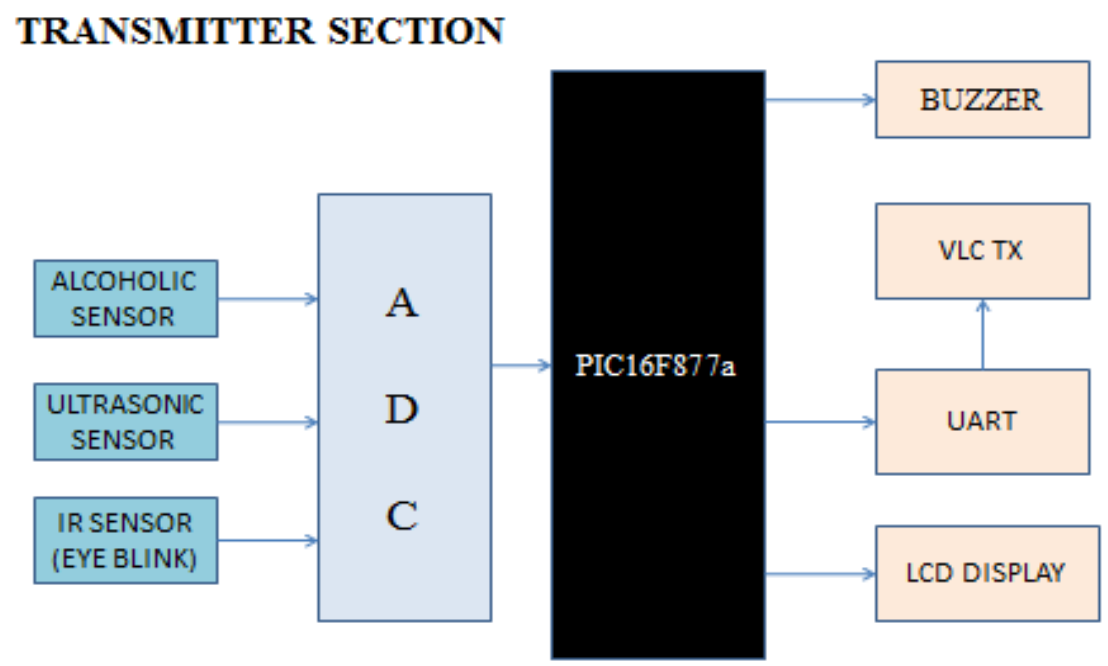

Figure 1. Transmitter section for VANET using Li-Fi.

\section{RECEIVER SECTION}

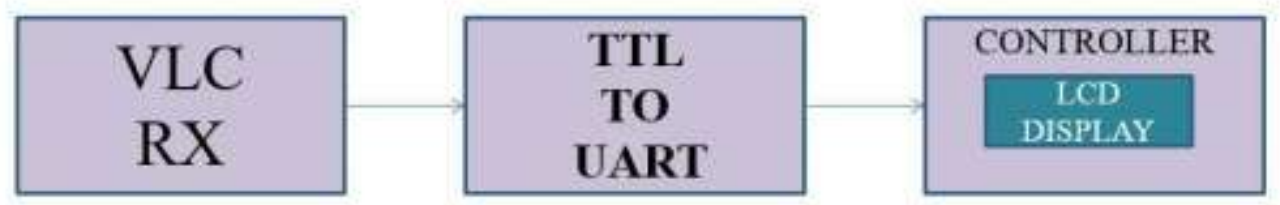

Figure 2. Receiver section for VANET using Li-Fi

\section{Methodology}

The sensor gives its data to the Analog to Digital Convertor (ADC) to convert the analog values to digital values. The microcontroller stores these values and transmitsthedata to the UART (Universal Asynchronous Receiver /Transmitter) which used for serial communication between the transmitter and receiver. The UART is one of the most important tools used when debugging is serial input or output. Serial is very easy to implement, and it allows you to send/receive any data you need from your microcontroller to a computer's serial port so it can be viewed using a terminal emulator for simulation purpose. This method of serial communication is sometimes referred to as a TTL serial (transistor-transistor logic). Asynchronous transmission allows data to be transmitted without the sender having to send a clock signal to the receiver. The buzzer circuit is switched on when the sensor detects any obstacles or if the person has consumed alcohol or closure of eyes is being detected by the eye blink sensor. The VLC transmitter transmits the binary data to the VLC receiver and it's been given to the TTL(Transistor Transistor Logic) to UART and been displayed in the LCD display. 


\section{Conclusion}

The road accidents are rapidly increasing day by day. One of the main reason for road accidents is caused due to tiredness of driver and eventuallythey fall asleep. In this project an effective eye movementdetection system has been devised wherein the state of theeyes has been continuously monitored using the eyeblink sensor.If found drowsy a signal is sent to the microcontroller which will turn ON the buzzer circuit to alert the driver.The alcohol sensor is used detects if the person driving the vehicle has consumedalcohol, if detected, then a signal is being sent to the microcontroller which transmit the signal to the neighboring vehicle using $\mathrm{Li}-\mathrm{Fi}$ and at the same time the relay unit is turned off. Due to this the ignition of the vehicle is deactivated. The ultrasonic sensor is used to measure the distance between vehicles or any obstacles to warn the driver of any dangers that may lie ahead on the road.This method has proven to be a cheaper and an effective method for accident prevention.

\section{Acknowledgement}

It is our immense pleasure to work on this Project, Design of Vehicle to Vehicle Data Transmission for IntelligentTransportation System using Li- Fi Technology. I offer my sincere thanks to our guide Prof.Bildass Santhosam. Iwho have affectionately encouragedus to work on the subject and gave his valuable guidance time to time.

\section{References}

[1]. O. Andrisano, R. Verdone, and M.Nakagawa, "Intelligent transportation systems: The role of third generation mobile radio networks,” IEEE Commun. Mag., vol. 38, no. 9, pp. 144-151, Sep. 2000.

[2]. V. Kumar, S. Mishra, and N. Chand, "Applications of VANETs: Present \&future,” Commun. Netw., vol. 5, no. 1B, pp. 12-15, 2013

[3]. Alin-M. Cailean, Barthélemy Cagneau, Luc Chassagne, Suat Topsu, "Design and implementation of a visible light communications system for vehicle applications", November 2013.

[4]. Shubham Chatterjee, Shalabh Agarwal, "Scope and Challenges in Light Fidelity(LiFi) Technology in Wireless Data Communication", June 2015.

[5]. Mehboob raza haider, manoj mdongre, "Vehicle to vehicle communication using visible light communication technology".

[6]. Ricvehicledo Scopigno, Alessia Autolitano, "The Potential Benefits of On-Board Li-Fi for the Cooperation among Vehicles.

[7]. "Vehicle-to-Vehicle Wireless Communication Protocols for Enhancing Highway Traffic Safety," S. Biswas. R. Tatchikou, and F. Dion IEEE Comm., Vol. 44, no. 1, pp. 74-82, Jan.2006.

[8]. "The Vehicle Collision Warning System Based On Gps" Sehun Kim, Sunghyun Lee, Inchan Yoon, Mija Yoon And DoHyeun Kim 2011 First Acis/Jnu International Conference On Computers, Networks, Systems, And Industrial Engineering .

[9]. Y. Xiang, Z. Liu, R. Liu, W. Sun, and W. Wang, "GeoSVR: A map-based stateless VANET routing," Ad Hoc Netw., vol. 11, no. 7, pp. 2125-2135, Sep. 2013

[10]. C. Lochert et al., "A routing strategy for vehicular ad hoc networks in city environments," in Proc. IEEE Intell. Veh. System 